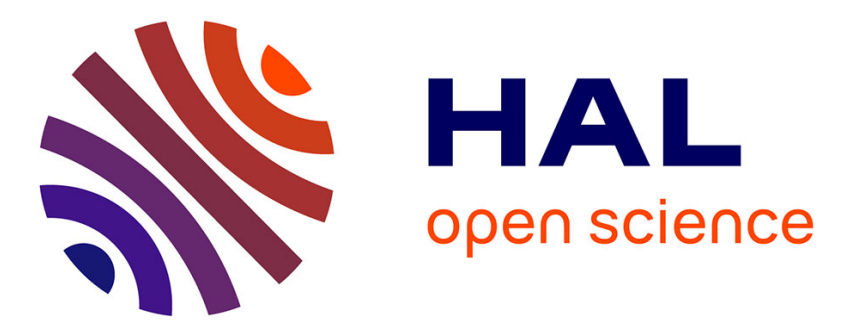

\title{
On Unitary Beamforming for MIMO Broadcast Channels
}

Sebastian Wagner, Stefania Sesia, Dirk Slock

\section{To cite this version:}

Sebastian Wagner, Stefania Sesia, Dirk Slock. On Unitary Beamforming for MIMO Broadcast Channels. IEEE International Conference on Communications, May 2010, Cape Town, South Africa. pp.1 - 5, 10.1109/ICC.2010.5502119 . hal-00675521

\section{HAL Id: hal-00675521 \\ https://hal.science/hal-00675521}

Submitted on 1 Mar 2012

HAL is a multi-disciplinary open access archive for the deposit and dissemination of scientific research documents, whether they are published or not. The documents may come from teaching and research institutions in France or abroad, or from public or private research centers.
L'archive ouverte pluridisciplinaire HAL, est destinée au dépôt et à la diffusion de documents scientifiques de niveau recherche, publiés ou non, émanant des établissements d'enseignement et de recherche français ou étrangers, des laboratoires publics ou privés. 


\section{On Unitary Beamforming for MIMO Broadcast Channels}

\author{
Sebastian Wagner \\ EURECOM, ST-Ericsson \\ Route des Lucioles, 635 \\ 06904 Sophia-Antipolis, France \\ Email: sebastian.wagner@eurecom.fr
}

\author{
Stefania Sesia \\ ST-Ericsson \\ Route des Lucioles, 635 \\ 06904 Sophia-Antipolis, France \\ Email: stefania.sesia@ stericsson.com
}

\author{
Dirk T. M. Slock \\ EURECOM \\ Route des Crêtes, B.P. 193 \\ 06904 Sophia-Antipolis, France \\ Email: dirk.slock@eurecom.fr
}

\begin{abstract}
In this paper we compare general unitary beamforming to constant modulus unitary beamforming in terms of achievable sum-rate for MIMO broadcast channels. We give a complete analysis of both schemes in the $2 \times 2$ multi-user MISO configuration. Furthermore, we study the regime of asymptotically high signal-to-noise ratio. For growing number of transmit antennas the sum-rate gap between both schemes is significantly increasing. Simulations are carried out that corroborate our analytical results.
\end{abstract}

\section{INTRODUCTION}

In the past years a lot of research has focused on characterizing the sum-rate capacities of the MIMO broadcast channel under different precoding strategies. It has been shown that the capacity achieving precoding scheme is based on the non-linear dirty-paper coding (DPC) technique [1]. But so far no efficient practical algorithm implementing the optimal DPC scheme has been found. Therefore, low complexity linear precoding or beamforming (BF) (e.g. zero-forcing BF) strategies have gained a lot of attention since they achieve a large portion of the channel capacity [2]-[4].

In this paper we compare two different types of unitary beamforming (UBF), general UBF and constant modulus UBF (CUBF). One advantage of applying UBF lies in the capability of the users to calculate their exact signal-tointerference and noise ratios (SINR) without knowledge of the channels/beamforming vectors of the interfering users. This is of practical importance since the SINR is an important feedback metric for the downlink resource allocation. The problem of the optimal parametrization of a UBF under perfect CSIT has been considered in [5] where an iterative optimization method based on successive Givens rotation has been proposed. However, in this contribution we will consider the steepest descent algorithm proposed in [6], since its implementation is straightforward.

The CUBF is motivated by practical constraints in the radio front-end. It is more efficient to have balanced transmit powers, since all power-amplifiers can be designed for the same dynamic range. Different additional amplitude variations of the transmit signal due to the precoding operation require a larger linearity region of the power amplifiers which renders them inefficient and more expensive. This is one of the reasons why codebooks containing unitary constant modulus matrices are chosen for single-user MIMO precoding, for instance in the LTE standard [7].

Usually the per-antenna power constraint (PAPC) is less stringent at the base-station. One reason is that the requirements on power amplifiers are less important at the basestation than for a mobile device. Furthermore, the basestation can allocate users, in e.g. frequency domain, that lead to approximately balanced antenna powers. However, in the uplink for example the constraint of equal antenna powers still persists, since in general the mobile device has less freedom in choosing different receivers for simultaneous transmission.

In the following, under the assumption of perfect CSIT, we study the achievable sum-rate of UBF and CUBF with and without power optimization. In particular we analyse and discuss the asymptotic SNR regime and compare both BF techniques. The main result is that CUBF with optimal power allocation outperforms UBF for low SNR but leads to a sumrate loss compared to UBF in the high SNR region. On the other hand the sum-rate of CUBF with equal power allocation saturates for high SNR.

The remainder of this paper is structured as follows: Section II introduces the system model. In Section III we briefly describe the parametrization of UBF and CUBF. Section IV studies the case of two users and two transmit antennas. Section V discusses the asymptotic SNR regimes of both UBF and CUBF. Section VI presents the simulation results. Finally, in Section VII we draw our conclusions.

Notation: In the following boldface lower-case and uppercase characters denote vectors and matrices, respectively. The operators $(\cdot)^{\mathrm{T}},(\cdot)^{\mathrm{H}}$ and $\operatorname{tr}(\cdot)$ denote transpose, conjugate transpose and the trace operator, respectively. The expectation is $E[\cdot]$ and $\operatorname{diag}(\mathbf{x})$ is a diagonal matrix with vector $\mathbf{x}$ on the main diagonal. The argument of a complex number $z$ is $\angle z$. The $N \times N$ identity matrix is $\mathbf{I}_{N}$.

\section{SYSTEM MODEL}

Consider a multi-user (MU) MISO downlink transmission scenario where an $M$-antenna transmitter communicates to $K$ non-cooperative single-antenna receivers. We denote the unitary beamforming matrix as $\mathbf{V}=\left[\mathbf{v}_{1}, \ldots, \mathbf{v}_{K}\right] \in \mathbb{C}^{M \times K}$, 
$\mathbf{V}^{\mathrm{H}} \mathbf{V}=\mathbf{V} \mathbf{V}^{\mathrm{H}}=\mathbf{I}$. The transmit signal $\mathbf{x}$ is formed as

$$
\mathbf{x}=\sum_{k=1}^{K} \sqrt{p_{k}} \mathbf{v}_{k} s_{k}
$$

where $p_{k}$ and $s_{k}\left(\left|s_{k}\right|^{2}=1\right)$ are the power and the information symbol of user $k$, respectively. Denote $\mathbf{R}_{\mathbf{x}}=E\left[\mathbf{x x}^{H}\right]$ the transmit signal covariance matrix and $\mathbf{W}=\operatorname{diag}\left(\left[p_{1}, \ldots, p_{K}\right]\right)$. The sum-power constraint imposes

$$
\operatorname{tr}\left(\mathbf{R}_{\mathbf{x}}\right)=\operatorname{tr}(\mathbf{W}) \leq P
$$

where $P$ is the total available transmit power. We consider narrow-band transmission. Hence for every channel use the received symbol vector reads

$$
\mathbf{y}=\mathbf{H x}+\mathbf{n}
$$

where $\mathbf{H}=\left[\mathbf{h}_{1}, \ldots, \mathbf{h}_{K}\right]^{\mathrm{H}} \in \mathbb{C}^{K \times M}$ is the channel matrix and $\mathbf{h}_{k} \in \mathbb{C}^{M \times 1}(k=1,2, \ldots, K)$ models the channel from the $M$-antenna transmitter to user $k$. The noise vector $\mathbf{n}$ is Gaussian distributed with $\mathbf{n} \sim \mathcal{C N}\left(0, \sigma^{2} \mathbf{I}_{K}\right)$ and we define the signal-to-noise ratio (SNR) as $\mathrm{SNR}=P / \sigma^{2}$. Under unitary beamforming with equal power (EP) allocation $\left(p_{k}=P / M\right)$ the SINR $\gamma_{k}$ of user $k$ takes the form

$$
\gamma_{k}=\frac{\rho_{k}^{2}}{\beta_{k}+1-\rho_{k}^{2}}
$$

with $\beta_{k}=\frac{M \sigma^{2}}{P\left\|\mathbf{h}_{k}\right\|_{2}^{2}}, \rho_{k}=\left|\overline{\mathbf{h}}_{k}^{\mathrm{H}} \mathbf{v}_{k}\right| \in[0,1]$ and $\overline{\mathbf{h}}_{k}=\frac{\mathbf{h}_{k}}{\left\|\mathbf{h}_{k}\right\|}$. The achievable sum-rate $R_{\text {sum }}$ of the system is given by

$$
R_{\text {sum }}(\mathbf{V})=\sum_{k=1}^{K} \log \left(1+\gamma_{k}\right) \quad[\text { nats } / \mathrm{s} / \mathrm{Hz}] .
$$

From the objective function (5) it follows that for any diagonal unitary matrix $\mathbf{D}=\operatorname{diag}\left(\left[e^{j \phi_{1}}, \ldots, e^{j \phi_{K}}\right]\right), \phi_{i} \in[0,2 \pi)$ we have

$$
R_{\text {sum }}(\mathbf{V})=R_{\text {sum }}(\mathbf{V D}) \text {. }
$$

Thus, it is always possible to dephase the first row of $\mathbf{V}$. We therefore further assume that the first row contains only real values.

\section{Parametrization of UBF AND CUBF}

In this section we describe how to parametrize the UBF and CUBF under sum-rate maximization.

\section{A. Optimization of $U B F$}

The optimization problem can be stated as follows

$$
\begin{gathered}
\mathbf{V}^{\star}=\underset{\mathbf{V}}{\arg \max }\left\{R_{\text {sum }}(\mathbf{V})\right\} \\
\text { s.t. } \quad \mathbf{V}^{\mathrm{H}} \mathbf{V}=\mathbf{I}_{K} .
\end{gathered}
$$

This is a nonconvex optimization problem with nonlinear constraints. In [5] this problem has been approached via an iterative algorithm based on successive Givens rotations. However, in this contribution we use the self-tuning Riemannian steepest descent algorithm given in Table II of [6] to solve the optimization problem in (7). Define the element $(i, j)$ of the gradient matrix $\boldsymbol{\Gamma}=\nabla_{\mathrm{V}} R_{\text {sum }}$ as

$$
[\boldsymbol{\Gamma}]_{i j}=\frac{\partial R_{\text {sum }}}{\partial\left[\mathbf{V}^{*}\right]_{i j}} .
$$

The gradient matrix $\Gamma$ of the cost function in (5) is given by

$$
\begin{aligned}
\boldsymbol{\Gamma} & =\left[c_{1} \mathbf{h}_{1} \mathbf{h}_{1}^{\mathrm{H}} \mathbf{v}_{1}, \ldots, c_{K} \mathbf{h}_{K} \mathbf{h}_{K}^{\mathrm{H}} \mathbf{v}_{K}\right] \\
\text { with } \quad c_{k} & =\left(\beta_{k}+1-\rho_{k}^{2}\right)^{-1} .
\end{aligned}
$$

The gradient direction in Riemannian space is defined as [6]

$$
\mathbf{G}=\boldsymbol{\Gamma} \mathbf{V}^{\mathrm{H}}-\mathbf{V} \Gamma^{\mathrm{H}} .
$$

Following the gradient $\mathbf{G}$ the algorithm guaranties to converge to a local extremum.

\section{B. Optimization of CUBF}

In this section we give a brief summary of the CUBF parametrization. In general the CUBF belongs to the set of normalized complex Hadamard matrices of size $M$ denoted $\mathcal{H}_{M}$ and defined as [8]

$$
\mathcal{H}_{M}=\left\{\mathbf{A}^{\mathrm{H}} \mathbf{A}=\mathbf{I}_{M}: \mathbf{A} \in \mathbb{C}^{M \times M} \text { and }\left|a_{i j}\right|^{2}=\frac{1}{M}\right\}
$$

with $i, j \in\{1, \ldots, M\}$. To describe the set $\mathcal{H}_{M}$ it is convenient, for our purpose of beamforming under sum-rate maximization, to divide $\mathcal{H}_{M}$ into equivalence classes. The equivalence relation is defined as follows

Definition 1: [8], The complex Hadamard matrices $\{\mathbf{A}, \tilde{\mathbf{A}}\} \in \mathcal{H}_{M}$ are equivalent, written $\mathbf{A} \cong \tilde{\mathbf{A}}$, iff there exist diagonal unitary matrices $\mathbf{D}_{\mathrm{r}}, \mathbf{D}_{\mathrm{c}}$ and permutation matrices $\mathbf{P}_{\mathrm{r}}, \mathbf{P}_{\mathrm{c}}$ such that ${ }^{1}$

$$
\mathbf{A}=\mathbf{D}_{\mathrm{r}} \mathbf{P}_{\mathrm{r}} \tilde{\mathbf{A}} \mathbf{P}_{\mathrm{c}} \mathbf{D}_{\mathrm{c}} .
$$

There are $M$ ! row and column permutations. The corresponding equivalence class $\mathbf{Q}_{M}(\mathbf{A})$ of $\mathbf{A} \in \mathcal{H}_{M}$ is

$$
\mathbf{Q}_{M}(\mathbf{A})=\left\{\mathbf{B} \in \mathcal{H}_{M} \mid \mathbf{A} \cong \mathbf{B}\right\} .
$$

The set of equivalence classes $\mathcal{H}_{M} / \cong$ is denoted $\mathcal{G}_{M}$.

Thus the CUBF can be parametrized using (14) and the equivalence classes (15). The complete set of equivalence classes $\mathcal{G}_{M}$ is only known for $M<6$. The equivalence classes $\mathcal{G}_{2}=\mathbf{Q}_{2}\left(\mathbf{F}_{2}\right)$ and $\mathcal{G}_{4}=\mathbf{Q}_{4}\left(\mathbf{Q}_{4}^{o}(\theta)\right)$ are particularly relevant in following for derivations and simulations. We have

$$
\mathbf{F}_{2}=\left[\begin{array}{cc}
1 & 1 \\
1 & -1
\end{array}\right]
$$

and $\mathcal{G}_{4}=\mathbf{Q}_{4}\left(\mathbf{Q}_{4}^{o}(\theta)\right)$ with $\theta \in\left[\frac{\pi}{2}, \frac{3}{2} \pi\right)^{2}$ where

$$
\mathbf{Q}_{4}^{o}(\theta)=\left[\begin{array}{cccc}
1 & 1 & 1 & 1 \\
1 & -1 & e^{j \theta} & -e^{j \theta} \\
1 & 1 & -1 & -1 \\
1 & -1 & -e^{j \theta} & e^{j \theta}
\end{array}\right]
$$

\footnotetext{
${ }^{1}$ In this definition transposition and complex conjugate are excluded since they are meaningless in the application of beamforming

${ }^{2}$ Since $\mathbf{Q}_{4}^{o}(\theta) \cong \mathbf{Q}_{4}^{o}(\theta+\pi)$
} 
A recent catalog of known equivalence classes can be found in [8]. Under sum-rate maximization and invariance (6) the CUBF takes the form

$$
\mathbf{V}^{\mathrm{CUBF}}=\frac{1}{\sqrt{M}} \mathbf{D}_{\mathrm{r}} \mathbf{P}_{\mathrm{r}} \mathbf{B}_{l} \mathbf{P}_{\mathrm{c}}
$$

where $\mathbf{D}_{\mathrm{r}}=\operatorname{diag}\left(\left[1, e^{j \varphi_{1}}, \ldots, e^{j \varphi_{M-1}}\right]\right)$ with $\varphi_{i} \in[0,2 \pi), i=$ $\{1, \ldots, M-1\}$ and $\mathbf{B}_{l}$ is a representative element of the $l$ th equivalence class.

To maximize the sum-rate (5) under equal power allocation we have to solve the following optimization problem

$$
\left\{\mathbf{D}_{\mathrm{r}}^{\star}, \mathbf{B}_{l}^{\star}, \mathbf{P}_{\mathrm{c}}^{\star}, \mathbf{P}_{\mathrm{r}}^{\star}\right\}=\underset{\mathbf{D}_{\mathrm{r}}, \mathbf{B}_{l}, \mathbf{P}_{\mathrm{c}}, \mathbf{P}_{\mathrm{r}}}{\arg \min }\left\{\prod_{k=1}^{K}\left(1+\beta_{k}-\rho_{k}^{2}\right)\right\} .
$$

An iterative approach to solve (19) has been proposed in [9] The algorithm optimizes the angels $\varphi_{i}, \theta$ successively until convergence to a local optimum. The optimal combination of row and column permutations has to be found exhaustively. To reduce the computational complexity due to the exhaustive search we will optimize the BF vectors in a greedy fashion. That is, we align one $\mathrm{BF}$ vector to the user with largest channel 1 -norm and then add the remaining users successively, such that the sum-rate is increasing. If none of the remaining users leads to an increase in sum-rate, the algorithm is terminated.

If an unequal power allocation is applied, the general SINR expression for linear precoding has to be used and the optimization problem takes the more general form

$$
\begin{aligned}
& \left\{\mathbf{D}_{\mathrm{r}}^{\star}, \mathbf{B}_{l}^{\star}, \mathbf{P}_{\mathrm{c}}^{\star}, \mathbf{P}_{\mathrm{r}}^{\star}\right\}= \\
& \underset{\mathbf{D}_{\mathrm{r}}, \mathbf{B}_{l}, \mathbf{P}_{\mathrm{c}}, \mathbf{P}_{\mathrm{r}}}{\arg \max }\left\{\sum_{k=1}^{K} \log \left(1+\frac{p_{k}\left|\mathbf{h}_{k}^{\mathrm{H}} \mathbf{v}_{k}\right|^{2}}{\sum_{\substack{i=1 \\
i \neq k}}^{K} p_{i}\left|\mathbf{h}_{k}^{\mathrm{H}} \mathbf{v}_{i}\right|^{2}+\sigma^{2}}\right)\right\} .
\end{aligned}
$$

The solution to the optimization problem (20), is a straightforward extension to the one with EP allocation in (19).

Since the joint optimization of BF vectors $\mathbf{v}_{k}$ and powers $p_{k}$ is intractable, we consider the two problems separately. Thus, with the cost function in (20) the powers are optimized for fixed $\mathrm{BF}$ vectors. Since the cost function is not convex in the $p_{k}$, we find an approximate solution using a standard interior point method [13]. More precisely, we eliminate the linear constraint (2) [13, Section 4.2.4] and introduce a logarithmic barrier function $\sum_{k=1}^{K} \log \left(p_{k}\right)$ to satisfy $p_{k} \in \mathbb{R}_{+}$. Subsequently we apply the barrier-method where we utilize Newton's method to compute a local optimum for the unconstrained optimization problem.

\section{AnALYSIS OF UBF AND CUBF FOR $K=M=2$}

In order to gain some insight on the underlying mathematical structure of the optimization problems we consider the case of two transmit antennas and two users.

\section{A. $U B F$}

The unitary matrix $\mathbf{U} \in S U(2)$ of dimension $M=2$ has $M^{2}-1=3$ real independent parameters and takes the form

$$
\mathbf{U}\left(z_{1}, z_{2}\right)=\left[\begin{array}{cc}
z_{1} & z_{2} \\
-z_{2}^{*} & z_{1}^{*}
\end{array}\right] \quad \text { s.t. } \quad\left|z_{1}\right|^{2}+\left|z_{2}\right|^{2}=1
$$

with $z_{1}, z_{2} \in \mathbb{C}$. Based on (6) we can dephase the first row of $\mathbf{U}$ by setting $\phi_{1}=-\angle z_{1}$ and $\phi_{2}=-\angle z_{2}$. Together with $\left|z_{1}\right|=\cos \alpha$ and $\left|z_{2}\right|=\sin \alpha$, satisfying the linear constraint in (21), we obtain

$$
\mathbf{V}^{\mathrm{UBF}}(\alpha, \delta)=\left[\begin{array}{cc}
\cos \alpha & \sin \alpha \\
\sin \alpha e^{j \delta} & -\cos \alpha e^{j \delta}
\end{array}\right]
$$

where $\delta=\pi-\left(\angle z_{1}+\angle z_{2}\right) \in(0,2 \pi]$ and $\alpha \in(0, \pi]^{3}$. Thus, for the purpose of beamforming there remain 2 real independent parameters $\alpha$ and $\delta$ for the sum-rate optimization.

Finding the optimal parameters $\alpha^{\star}$ and $\delta^{\star}$ has been covered in [5]. The solution involves finding the real roots of a polynomial $P_{\alpha}(\delta)$ and $P_{\delta}(\alpha)$ of order 4 , for both $\alpha$ and $\delta$, respectively. Since the roots of both polynomials depend on either $\alpha$ or $\delta$, the joint local optimum $\left(\alpha^{\star}, \delta^{\star}\right)$ has to be computed iteratively. That is, by alternating between $\alpha_{k}^{\star}\left(\delta_{k-1}\right)$ and $\delta_{k}^{\star}\left(\alpha_{k-1}\right)$ until convergence.

\section{B. CUBF with Equal Power Allocation}

In the case of $M=2$ the permutations $\mathbf{P}_{\mathrm{r}}$ and $\mathbf{P}_{\mathrm{c}}$ have no impact on the sum-rate optimization, thus from (18) we have

$$
\mathbf{V}^{\mathrm{CUBF}}(\delta)=\frac{1}{\sqrt{2}}\left[\begin{array}{cc}
1 & 1 \\
e^{j \delta} & -e^{j \delta}
\end{array}\right]
$$

where $\delta \in(0,2 \pi]$. Thus there is only one real independent parameter left for the sum-rate optimization. From (22) we observe that for $\alpha=\frac{\pi}{4}$ the general UBF has uni-modular entries and thus coincides with the CUBF${ }^{4}$, i.e. $\mathbf{V}^{\mathrm{CUBF}}(\delta)=$ $\mathbf{V}^{\mathrm{UBF}}\left(\frac{\pi}{4}, \delta\right)$. Hence, the optimization of $\delta$ involves finding the real roots of $P_{\delta}\left(\frac{\pi}{4}\right)$. Consequently, for CUBF with equal power allocation (CUBF-EP), the optimal angle $\delta^{\star}$ can be computed exactly leading to the global optimal $\mathbf{V}^{\mathrm{CUBF}}\left(\delta^{\star}\right)$. Note that in general, i.e. $\alpha \neq \frac{\pi}{4}$, we have $\delta_{\mathrm{UBF}}^{\star} \neq \delta_{\mathrm{CUBF}}^{\star}$.

From (23), it is clear that perfect alignment, i.e. $\rho_{k}=1$, can not be achieved with the CUBF (only when all channel gains are equal). Consequently, the sum-rate $R_{\text {sum }}\left(\mathbf{V}^{\mathrm{CUBF}}\right)$ is interference-limited for high SNR. Also, at high SNR it is not optimal for CUBF-EP to align to the user with largest channel 1-norm.

\section{CUBF with Optimal Power Allocation}

In addition to the beamforming matrix (23) we can also optimize the power $p_{k}$ allocated to user $k,=1,2$. This scheme is called CUBF-OP. The SINR (4) takes the form

$$
\gamma_{k}=\frac{p_{k} \rho_{k}^{2}}{\tilde{\beta}_{k}+p_{3-k}\left(1-\rho_{k}^{2}\right)}
$$

\footnotetext{
${ }^{3}$ since $\rho_{k}^{2}(\alpha)$ has period $\pi$.

${ }^{4}$ note also that $\alpha^{\star}=\frac{\pi}{4}$ for equal channel gains i.e. $\left|h_{i j}\right|^{2}=c$.
} 
where $\tilde{\beta}_{k}=\frac{\sigma^{2}}{\left\|\mathbf{h}_{k}\right\|_{2}^{2}}, p_{2}=P-p_{1}$ and $p_{1} \in(0, P)$. Calculating $p_{1}^{\star}$ such that (5) is maximized for a given $\delta$ implies finding the zeros of the rational function $f\left(p_{1}\right)=r\left(p_{1}\right) / q\left(p_{1}\right)$ s.t. $p_{1}^{\star} \in(0, P)$, where $r\left(p_{1}\right)$ and $q\left(p_{1}\right)$ are polynomials of order 2 and 4, respectively. Since (24) is in general not convex in $(0, P)$ it can not be assured that the zeros of $f\left(p_{1}\right)$ lie in $(0, P)$. Consequently, if there are no real solutions in $(0, P)$ the solution must be one of the border points i.e. either $p_{1}^{\star}=0$ or $p_{1}^{\star}=P$.

Note that for asymptotically high SNR i.e. $\beta_{k} \rightarrow 0$, (24) is maximized for either $p_{1}^{\star}=0$ or $p_{1}^{\star}=P$.

\section{DISCUSSION}

In this section we discuss some aspects of UBF, CUBF-EP and CUBF-OP for the low and high SNR regions.

\section{A. Low SNR Region}

It is well known that at low SNR the optimal strategy is to perform TDMA [10] to the user with largest channel 2norm. The behavior of UBF in the low SNR region has been investigated in [11]. Since UBF can never switch off a user completely its performance is inferior to TDMA for low SNR. The same is true for CUBF-EP, but the performance loss is more significant than for UBF.

Since CUBF can only adapt to the phases of the user channels it is optimal to align the CUBF to the user $n$ with largest channel 1-norm. This is identical to equal gain transmission [12]. Only CUBF-OP is able to perform equal gain transmission to the user with largest channel 1-norm. Still its performance is slightly inferior to TDMA since a perfect alignment cannot be accomplished.

\section{B. High SNR Region}

All three schemes are interference limited for high SNR. Therefore it is optimal for UBF to align one BF vector perfectly to the channel with the largest 2-norm since this user experiences no interference and the sum-rate scaling is equal or superior to that of TDMA, [5].

To avoid sum-rate saturation, the CUBF-OP performs (as for low SNR) equal gain transmission to the user with largest channel 1-norm and switches off all remaining users. Therefore at high SNR the sum-rate of CUBF-OP can be written as

$$
R_{\text {sum }}=\log (\mathrm{SNR})-\log (M)+E\left[\log \left(\max \|\mathbf{h}\|_{1}^{2}\right)\right] .
$$

The multiplexing gain is defined as

$$
m=\lim _{P \rightarrow \infty} \frac{E\left[R_{\mathrm{sum}}\right]}{\log P} .
$$

For CUBF-OP we have

$m^{\mathrm{CUBF}-\mathrm{OP}}=\lim _{P \rightarrow \infty} \frac{E\left[\log \left(1+\frac{P}{M \sigma^{2}}\left\|\mathbf{h}_{n}\right\|_{1}^{2}\right)\right]}{\log P}=1 \leq m^{\mathrm{UBF}}$

where $\mathbf{h}_{n}$ is the channel with maximum 1-norm. Hence, UBF as well as CUBF-OP achieve at least a multiplexing gain of one. But there is a power offset between both schemes. This

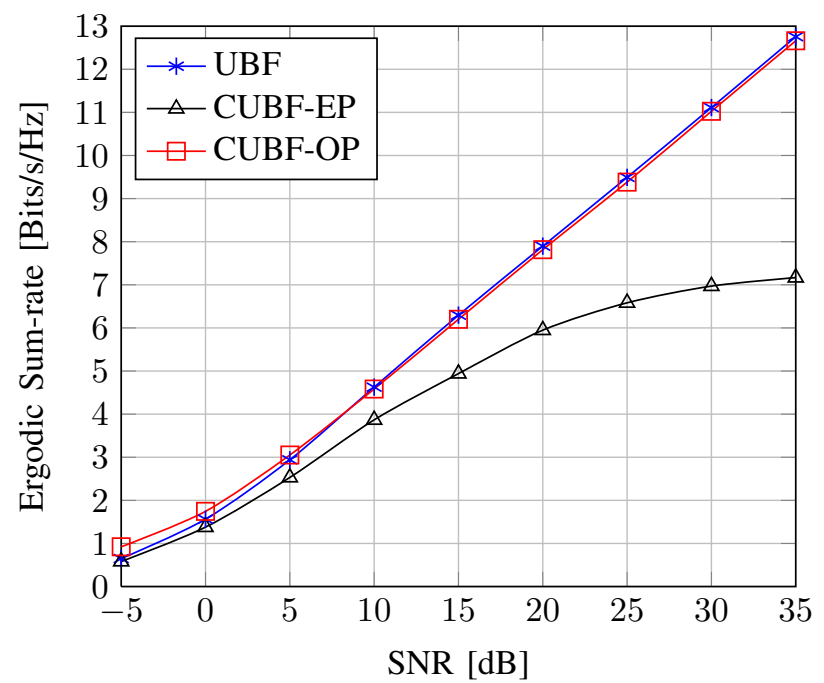

Fig. $1 . \quad 2 \times 2$ MU-MISO, Ergodic Sum-rate vs. SNR

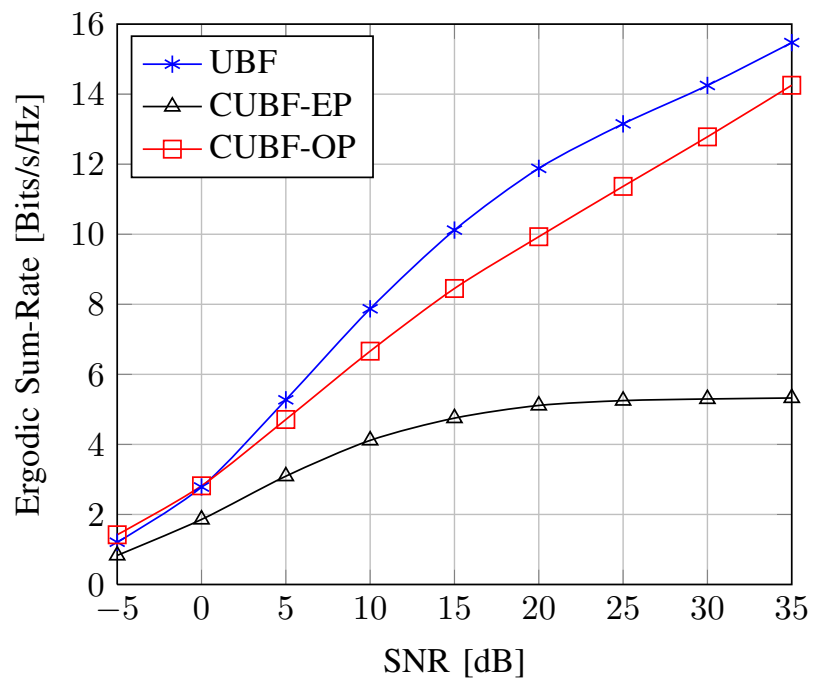

Fig. 2. $4 \times 4$ MU-MISO, Ergodic Sum-rate vs. SNR

power offset increases with $M$ since UBF offers more degrees of freedom as CUBF-OP with growing $M$.

It is also clear that the sum-rate saturates for CUBF-EP, since neither users can be switched off, nor perfect alignment can be achieved. Hence, this scheme has no means to combat inter-user interference effectively.

\section{Simulation AND Results}

In this section we compare UBF, CUBF-EP and CUBFOP in terms of ergodic sum-rate i.e. $E_{\mathbf{H}}\left[R_{\text {sum }}(\mathbf{V})\right]$, over independent Rayleigh fading channels.

Figure 1 shows the performance in a $2 \times 2$ MU-MISO configuration. As discussed in Section $\mathrm{V}$ we observe that in the low SNR regime, CUBF-OP achieves slightly higher sum-rate than UBF and CUBF-EP. For high SNR, UBF and CUBF-OP perform similar although UBF is slightly superior due to the 
perfect alignment. As expected, the sum-rate of CUBF-EP is saturating at high SNR.

Figure 2 depicts the performance of a $4 \times 4$ MU-MISO setup. It can be observed that, except for low SNR, UBF significantly outperforms CUBF-OP. For high SNR both UBF and CUBFOP have the same slope although there is a significant poweroffset between the two schemes.

Moreover, the sum-rate saturation level of CUBF-EP is lower for $M=4$ than for $M=2$. This is due to the fact that the degrees of freedom of CUBF-EP scale significantly slower with $M$ than the degrees of freedom of the channel. Thus, the CUBF-EP is increasingly maladjusted to the channel and consequently the interference level is rising.

\section{CONCLUSION}

This contribution compared UBF, CUBF-EP and CUBFOP in terms of achievable sum-rate in the MISO broadcast channel. In particular we analyzed the performance of both schemes in the $2 \times 2$ MU-MISO downlink channel. In this configuration UBF and CUBF-OP perform very similar although CUBF-OP slightly outperforms UBF for low SNR and UBF is slightly superior in the high SNR region. If the number of transmit antennas and user increase UBF is significantly superior to CUBF-OP due to the additional degrees of freedom in the parametrization. But both schemes achieve a multiplexing gain of one.

\section{REFERENCES}

[1] H. Weingarten, Y. Steinberg, and S. Shamai, "The Capacity Region of the Gaussian Multiple-Input Multiple-Output Broadcast Channel," IEEE Trans. Inf. Theory, vol. 52, no. 9, pp. 3936-3964, 2006.

[2] G. Caire and S. Shamai, "On the Achievable Throughput of a Multiantenna Gaussian Broadcast Channel," IEEE Trans. Inf. Theory, vol. 49, no. 7, pp. 1691-1706, 2003.

[3] C. Peel, B. Hochwald, and A. Swindlehurst, "A Vector-Perturbation Technique for Near-Capacity Multiantenna Multiuser CommunicationPart I: Channel Inversion and Regularization," IEEE Trans. Commun., vol. 53, no. 1, pp. 195-202, 2005.

[4] A. Wiesel, Y. Eldar, and S. Shamai, "Zero-forcing Precoding and Generalized Inverses," IEEE Trans. Signal Process., vol. 56, no. 9, p. 4409, 2008.

[5] R. de Francisco and D. T. Slock, "An Iterative Optimization Method for Unitary Beamforming in MIMO Broadcast Channels," in Proc. of 45th Allerton Conf. on Communication, Control and Compuing, Sep. 2007, pp. $360-367$.

[6] T. Abrudan, J. Eriksson, and V. Koivunen, "Steepest Descent Algorithms for Optimization Under Unitary Matrix Constraint," IEEE Trans. Signal Process., vol. 56, no. 3, pp. 1134-1147, 2008.

[7] S. Sesia, I. Toufik, and M. Baker, Eds., LTE, The UMTS Long Term Evolution: From Theory to Practice, 1st ed. Wiley \& Sons, 2009.

[8] W. Tadej and K. Życzkowski, "A Concise Guide to Complex Hadamard Matrices," Open Systems \& Information Dynamics, vol. 13, no. 2, pp. 133-177, 2006.

[9] S. Wagner, S. Sesia, and D. Slock, "Unitary Beamforming Under Constant Modulus Constraint in MIMO Broadcast Channels," in Signal Processing Advances in Wireless Communications, 2009. SPAWC '09. IEEE 10th Workshop on, Jun. 2009, pp. 151-155.

[10] N. Jindal and A. Goldsmith, "Dirty-paper coding versus TDMA for MIMO Broadcast channels," IEEE Trans. Inf. Theory, vol. 51, no. 5, pp. 1783-1794, 2005.

[11] R. de Francisco, M. Kountouris, D. Slock, and D. Gesbert, "Orthogonal Linear Beamforming in MIMO Broadcast Channels," in Proceedings of IEEE Wireless Communications and Networking Conference (WCNC07), 2007, pp. 1210-1215.
[12] D. J. Love and R. W. H. Jr., "Equal Gain Transmission In Multiple-Input Multiple-Output Wireless Systems," IEEE Trans. Commun., vol. 51, no. 7, pp. 1102-1110, 2003.

[13] S. P. Boyd and L. Vandenberghe, Convex Optimization. Cambridge University Press, 2004. 